\section{A Giant Ossified Chronic Subdural Hematoma}

Sir,

A 49-year male was admitted to this hospital with complaints of progressive headache for more than one month. The initial computed tomography (CT) and magnetic resonance imaging (MRI) of brain showed a calcified subdural mass covering the right hemisphere (Figures 1A-D). The subdural mass had a low signalintense outer layer and a mixed signal-intense centre on both $T 1$ and T 2 weighted imaging (Figures $1 A-B$ ), and had hyperdense outer rims and a heterogeneous density central part on CT (Figure 1C). Right frontotemporoparietal craniotomy was performed. When the bone flap was turned down, a mild reactive hyperplasia of its inner plate was noticed. The dura mater was thin and severely adherant to the mass, not easily separable. The dura mater was dissected back, and a crust-like subdural mass was exposed. There was no adhesion between the mass and the arachnoid membrane. Careful dissection was performed, and the mass was completely removed. On opening the mass, there was some gray to red brown, "bean paste" inside, mixed with some calcific granules. Pathological examination revealed the "bean paste" to be composed of necrotic material with hemorrhage and calcification (Figure 1E), with other areas of the mass showing typical appearance of bone (Figure 1F). CT scan done 1.5 months after the operation showed the hematoma to be cleared (Figure 1D). The patient had a good recovery.

Ossification of chronic subdural hematoma is rare, ${ }^{1}$ and may be caused by head injury, surgeries, intracranial infections, and alcoholism. ${ }^{2}$ Vascular, metabolic and some local factors may play a part in this process. ${ }^{3,4}$ An ossified crust-like mass covering the hemisphere can be found on CT and MRI. Surgical treatment is effective. Surgical incision and skull bone flap should be large enough. Full exposure of the edge of the ossified hematoma is necessary for its removal in toto. It is essential to try to avoid cutting up the mass during the operation, because the contents of the mass can stimulate the brain tissue and vessels and cause postoperative fever, headache, and epilepsy. Most of such masses are not densely adherant to the arachnoid membrane and cerebral cortex. As long as they are carefully dissected, it is not difficult to take them out completely.

It is not difficult to make a correct preoperative diagnosis of ossified chronic subdural hematoma by CT and MRI. Proper surgical treatment is the recommended course of treatment for symptomatic patients, and most patients have a good outcome.

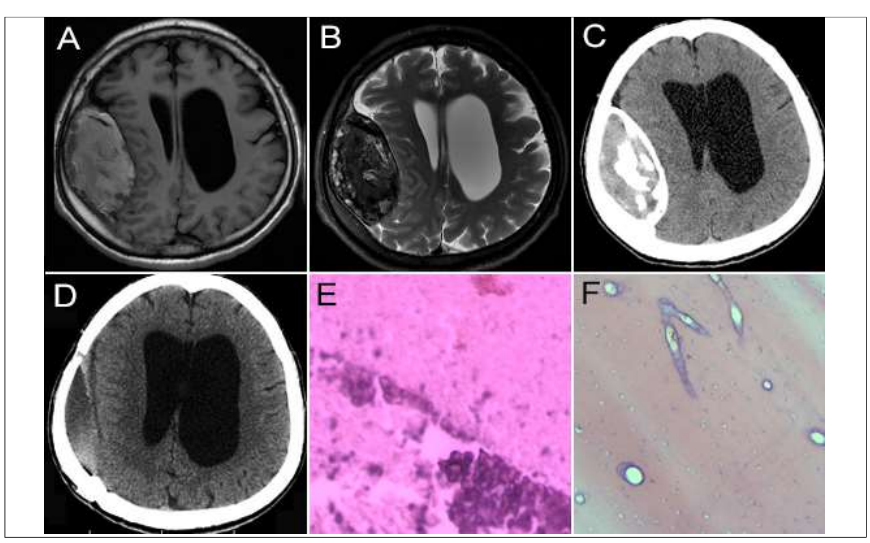

Figure 1: (A) The initial T1-weighted imaging of the patient. (B) The initial T2-weighted imaging of the patient. (C) The initial CT of the patient. (D) The postoperative CT scan showing a subdural effusion in the hematoma cavity. (E) Histopathology of the "bean paste", showing necrosis, hemorrhage, and calcification (hematoxylin-eosin, $\times 40$ ). (F) Histopathology of the mass showing the typical appearance of bone (hematoxylin-eosin, $\times 40$ ).

\section{CONFLICT OF INTEREST:}

Authors declared no conflict of interest.

\section{AUTHORS' CONTRIBUTION:}

WT, XM, JZ: Substantial contributions to the conception or design of the work; or the acquisition, analysis, or interpretation of data for the work; drafting the work or revising it critically for important intellectual content; and final approval of the version.

\section{REFERENCES}

1. Siddiqui SA, Singh PK, Sawarkar D, Singh M, Sharma BS. Bilateral ossified chronic subdural hematoma presenting as diabetes insipidus: Case report and literature review. World Neurosurg 2017; 98:520-4.

2. Mori K, Maeda M. Surgical treatment of chronic subdural hematoma in 500 consecutive cases: Clinical characteristics, surgical outcome, complications, and recurrence rate. Neurol Med Chir 2001; 41:371-81.

3. Afra D. Ossification of subdural hematoma: Report of two cases. J Neurosurg 1961; 18:393-7.

4. Mclaurin RL, Malaurin KS. Calcifield subdural hematomas in childhood. J Neurosurg 1966; 24:648-55.

Wei Tian, Xin Meng and Jidian Zou

Department of Neurosurgery, Weihai Central Hospital, The Affiliated Hospital of Qingdao University Medical College, China

Correspondence to: Dr. Jidian Zou, Department of Neurosurgery, Weihai Central Hospital, The Affiliated Hospital of Qingdao University Medical College, China

E-mail:zoujidian@126.com

Received: December 05, 2018; Revised: February 28, 2019; Accepted: February 28, 2019

$$
\cdots \cdot \hat{r} \cdot \cdots
$$

